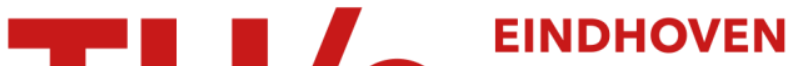 UNIVERSITY OF TECHNOLOGY
}

\section{Caging of a d-dimensional sphere and its relevance for the random dense sphere packing}

Citation for published version (APA):

Peters, E. A. J. F., Kollmann, M., Barenbrug, T. M. A. O. M., \& Philipse, A. P. (2001). Caging of a d-dimensional sphere and its relevance for the random dense sphere packing. Physical Review E-Statistical, Nonlinear, and Soft Matter Physics, 63(2), 021404-1/8. https://doi.org/10.1103/PhysRevE.63.021404

DOI:

10.1103/PhysRevE.63.021404

Document status and date:

Published: 01/01/2001

\section{Document Version:}

Publisher's PDF, also known as Version of Record (includes final page, issue and volume numbers)

\section{Please check the document version of this publication:}

- A submitted manuscript is the version of the article upon submission and before peer-review. There can be important differences between the submitted version and the official published version of record. People interested in the research are advised to contact the author for the final version of the publication, or visit the $\mathrm{DOI}$ to the publisher's website.

- The final author version and the galley proof are versions of the publication after peer review.

- The final published version features the final layout of the paper including the volume, issue and page numbers.

Link to publication

\section{General rights}

Copyright and moral rights for the publications made accessible in the public portal are retained by the authors and/or other copyright owners and it is a condition of accessing publications that users recognise and abide by the legal requirements associated with these rights.

- Users may download and print one copy of any publication from the public portal for the purpose of private study or research.

- You may not further distribute the material or use it for any profit-making activity or commercial gain

- You may freely distribute the URL identifying the publication in the public portal.

If the publication is distributed under the terms of Article $25 \mathrm{fa}$ of the Dutch Copyright Act, indicated by the "Taverne" license above, please follow below link for the End User Agreement:

www.tue.nl/taverne

Take down policy

If you believe that this document breaches copyright please contact us at:

openaccess@tue.nl

providing details and we will investigate your claim. 


\title{
Caging of a $d$-dimensional sphere and its relevance for the random dense sphere packing
}

\author{
E. A. J. F. Peters, ${ }^{1}$ M. Kollmann, ${ }^{2}$ Th. M. A. O. M. Barenbrug, ${ }^{1}$ and A. P. Philipse ${ }^{3, *}$ \\ ${ }^{1}$ Laboratory for Aero- and Hydrodynamics, Delft University of Technology, Rotterdamseweg 145, 2628 AL Delft, The Netherlands \\ ${ }^{2}$ Fakultät für Physik, Universität Konstanz, Postfach 5560, D 7750-Konstanz, Germany \\ ${ }^{3}$ Van 't Hoff Laboratorium for Physical and Colloid Chemistry, Debye Institute, Utrecht University, Padualaan 8 , \\ 3584 CH Utrecht, The Netherlands
}

(Received 25 July 2000; published 25 January 2001)

\begin{abstract}
We analyze the caging of a hard sphere (i.e., the complete arrest of all translational motions) by randomly distributed static contact points on the sphere surface for arbitrary dimension $d \geqslant 1$, and prove that the average number of uncorrelated contacts required to cage a sphere is $\langle N\rangle_{d}=2 d+1$. Computer simulations, which confirm this analytical result, are also used to model the effect of correlations between contacts that occur in real hard-sphere systems. Our analysis predicts an average coordination number of $4.79( \pm 0.02)$ for caged spheres, which agrees surprisingly well with the experimental coordination number for random sphere packings reported by Mason [Nature 217, 733 (1968)]. This result supports the physical picture that the coordination number in random dense sphere packings is primarily determined by caging effects. It also suggests that it should be possible to construct such packings from a local caging rule.
\end{abstract}

DOI: 10.1103/PhysRevE.63.021404

PACS number(s): 82.70.-y

\section{INTRODUCTION}

A "particle cage" is a very useful concept for the understanding of packed granular matter or dense colloidal particle systems. For example, hindered self-diffusion of colloidal spheres in a concentrated colloidal suspension can be seen as a sequence of "caging" events: a test sphere is temporarily trapped by a mobile cage of neighbor spheres, and eventually diffuses into another cage due to thermal fluctuations [1]. As the sphere concentration increases, the cages become less mobile, up to the point where the test sphere is permanently arrested by a cage of static neighbor spheres.

Such permanent caging of spheres will also occur in random dense sphere packings, prepared by rapid (on the diffusion time scale) sedimentation of colloid spheres [2]. These random packings or sphere glasses, with typical sphere volume fractions of $\phi \sim 0.64$, are instances of Bernal's random close sphere packing [3-6]. Other instances are the widely studied random packings of macroscopic spheres [7-21], where the jamming of spheres can also be seen as a caging effect.

The concept of a sphere cage is appealing, but still very qualitative. For example, one obvious question has not yet been answered satisfactorily: how many sphere contacts actually are needed to cage a test sphere in a system such as a random sphere packing? A tetrahedron of four neighbor sphere contacts will keep a test sphere in a mechanically stable position. In a random sphere system, however, neighbors need not form this tetrahedral configuration, so the average number of spheres required to form a cage must exceed four. The calculation of this average number (the "average cage size") is a complicated problem of statistical geometry, due to the correlations between contacts in a hard-sphere stacking. These correlations result from the fact that the

\footnotetext{
*Author to whom correspondence should be addressed.
}

neighbor spheres, which touch the test sphere, cannot interpenetrate each other.

To make a start with quantifying caging phenomena we have investigated a simple geometrical model for a static sphere cage, which completely and permanently arrests the sphere. In this reference model, neighbor spheres only experience hard-sphere excluded volume interaction with the central test sphere, whereas any interactions between the neighbors themselves are absent.

Our main result is that, within this approximation, the caging problem can be solved analytically, for arbitrary dimension $d \geqslant 1$. In our model we consider a single test sphere, with immobile point contacts randomly distributed on its surface, and investigate the probability that configurations of these static contacts block all translations of the test sphere in $d$ dimensions. We show that the average number $\langle N\rangle_{d}$ of such random contacts, which cage the sphere, increases linearly with the dimension as $\langle N\rangle_{d}=2 d+1$.

The caging of a sphere by random contacts was only solved earlier for a sphere in two dimensions (which is equivalent to the caging of a disc in a plane) [22]. For a three-dimensional (3D) sphere only numerical results for the average cage size have been reported $[23,24]$. The method from Ref. [22] for a 2D sphere is difficult to extend to higher dimensions. We have found a very convenient procedure to evaluate caging probabilities which is easy to generalize to higher dimensions. The procedure is based on regrouping all possible configurations of the $N$ contact points into equivalent subsets of a finite number of configurations. Every subset contains all information required for a calculation of the caging probability (for that particular $N$ and $d$ ). Therefore, it is not necessary to consider the total (infinite number) of all possible configurations.

We start in Sec. II with some definitions needed for the analysis in later sections. For clarity, but without loss of generality, we use terminology for the case of a sphere in $d=3$. The regrouping procedure is further explained in Secs. III and IV, and elaborated in Sec. V for the case $d=3$. The 


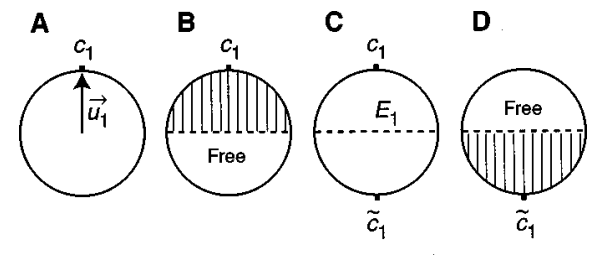

FIG. 1. (A) Direction vector $\vec{u}_{1}$ and contact $c_{1}$ on a sphere. (B) Contact $c_{1}$ forbids all direction vectors in shaded hemisphere. (C) Contact $c_{1}$ is reflected in the plane containing equator $E_{1}$ to its antipode $\widetilde{c}_{1}$. (D) This reflection turns the forbidden surface sector from $B$ into a free one, and vice versa.

generalization to arbitrary dimensions is made in Sec. VI. A comparison with simulations of sphere caging is made in Sec. VII. Simulations confirm the analytical result for random point constraints. In addition, we extended our simulations to the caging of a test sphere by spherical constraints with the same size as the central sphere, to model the effect of excluded-volume repulsions between the constraining spheres in a real hard-sphere system. The relevance of our findings regarding an interpretation of experimental results for coordination numbers in random sphere packings is discussed in Secs. VIII and IX.

\section{DEFINITIONS}

A configuration is defined as any distribution of $N$ constraints on a sphere surface. These constraints may be randomly placed contact points or spherical constraints (which are point constraints with an additional condition concerning the distance between them). A configuration is caging if the constraints are placed in such a way that the sphere cannot translate in any direction. The caging probability is the probability that a randomly chosen configuration is caging. Clearly, this probability depends on the dimensionality $d$ and on the number of constraints $N$.

Direction vectors $\vec{u}$ are vectors from the center of the sphere to its surface $S^{2}$. The sphere center can move only in the direction of a free direction vector, but cannot move in the direction of a forbidden direction vector. Thus a sphere is caged when it has no free direction vector. The sphere is noncaged when it has at least one free direction vector. A free point on $S^{2}$ is the end of a free direction vector. A free surface sector is a part of $S^{2}$ which contains free points only. (Likewise we can define forbidden points, forbidden segments, and forbidden surface sectors.) A contact is a pointlike obstacle by which we create a forbidden point on $S^{2}$. Let $c_{1}$ be such a contact at position $\vec{u}_{1}$ (see Fig. 1). The contact makes $\vec{u}_{1}$ a forbidden vector. But if $\vec{u}_{1}$ is forbidden by $c_{1}$, so is every other direction vector which has a component in the direction of $\vec{u}_{1}$. These forbidden vectors form a forbidden surface sector in the form of a hemisphere with $c_{1}$ at its pole. The other hemisphere is a free surface sector. The two hemispheres are separated by the equator (or great circle [25]) $E_{1}$ associated with contact $c_{1}$.

\section{REFLECTION SET $R$}

Using definitions from Sec. II we introduce the reflection set $R$ via a number of propositions about surface sectors.

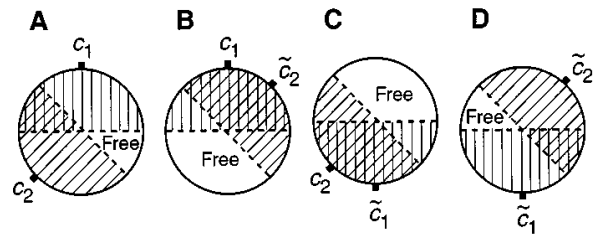

FIG. 2. The set $R$ consists of configurations of contacts which may or which may not be reflected. Each member of $R$ generates the whole set. Each surface sector is free only in one configuration of $R$.

Place $N$ contacts at fixed, random positions on $S^{2}$. The contacts produce $N$ equators which intersect each other; the probability that by this operation two equators coincide is zero. The intersecting equators form surface sectors (which are bounded by segments of these equators). The number of surface sectors only depends on the chosen values of $N$ and $d$. Clearly, a sector is forbidden when it is forbidden by at least one contact. For a free sector we therefore can state the following: (1) A sector is free if and only if it is free for all contacts.

In the foregoing we use the fact that for each (contact) point $c$ on $S^{2}$ there is only one unique great circle, which plays the role of the equator $E$, with $c$ as a pole. On the other hand, for any great circle there can be only two points of $S^{2}$ which are its poles [25]. In other words, every contact $c$ has a diametrically opposite or antipode point $\widetilde{c}$, formed by reflection of $c$ in the plane containing equator $E$ (the equator plane). We call this a contact reflection. This contact reflection changes surface points, free with respect to $c$, into forbidden ones, and vice versa. One consequence of a reflection is the following: (2) A free surface sector for contact $c$ is a forbidden surface sector for its antipode $\widetilde{c}$.

The (infinite) set of all possible configurations is divided in subsets $R$ as follows (see Fig. 2). Take any configuration from the infinite set. Each of the $N$ contact points in this configuration may be reflected to become its antipode. In this way a reflection set $R$ is generated containing $2^{N}$ different, but equivalent, configurations. These configurations are equivalent in the sense that they can all be transformed into each other by at most $N$ contact reflections. In other words, we can state the following: (3) Each member of a reflection set $R$ generates the whole set by contact reflections.

Since a contact $c$ and its antipode $\widetilde{c}$ necessarily share the same equator $E$, it follows that the partitioning of surface sectors by the $N$ equators is the same for all members of the same subset $R$. Nevertheless, even though a particular surface sector does not change in shape or position, the sector may be free in one configuration of $S$, and forbidden in another. There is, however, an important restriction: (4) Each surface sector in the reflection set $R$ is a free surface sector for one member of $R$ only.

Proposition (4) follows from the foregoing propositions. Let $a$ be a free surface sector in configuration $s$. The sector $a$ is free because it is free for all contact points [Proposition (1)]. The whole set $R$ can be generated from $s$ by contact reflections [Proposition (3)]. But every reflection turns $a$ into a forbidden sector [Proposition (2)]. Hence $a$ can only be 
free in the configuration $s$. The same reasoning applies to all other sectors in the reflection set $R$, which proves proposition (4).

We will now prove the following proposition: (5) For every member of $R$ there is at most one surface sector free. Let $a_{1}$ be a free surface sector in configuration $s$, and let $a_{2}$ be another surface sector in $s$. In going from $a_{1}$ to $a_{2}$ at least one equator (surface sector boundary) $E$ must be crossed. Let $c$ be the contact associated with $E$. Surface $a_{1}$ is free for all contact points [Proposition (1)]. Then by crossing $E$, the hemisphere of free directions associated with $c$ is left. Thus $a_{2}$ is forbidden (with respect to $c$ [propositions (1) and (2)]). As a result of propositions (4) and (5) we can finally conclude: (6) The number of surface sectors is equal to the number of noncaging members of $R$.

\section{CAGING PROBABILITY}

Let $A(N, d)$ be the total number of surface sectors produced by (the equators of) $N$ random contact points on a $d$ dimensional sphere. According to proposition (6), this number equals the number of noncaging configurations (i.e., configurations with one free surface sector) in the set $R$. Therefore the probability $C(N, d)$ that a member of $R$ does not cage a sphere is

$$
C(N, d)=A(N, d) / 2^{N} .
$$

All members of the infinite set of configurations of $N$ contact points are equivalent in the sense that each of them can be a member of one reflection set $R$ only. (A reflection of a contact point by definition only produces its antipode, and not any other contact position on $S^{2}$, which would be required to go from one reflection set $R$ to another.) Since each reflection set $R$ has the same fraction of noncaging members (for present $N$ and $d$ values), it follows that $C(N, d)$ is also the probability that $N$ contacts do not cage a $d$-dimensional sphere.

The number of contacts required to cage the sphere is called the cage size. The average cage size $\langle N\rangle_{d}$ follows from

$$
\langle N\rangle_{d}=\sum_{N=1}^{\infty} P(N, d) N,
$$

where $P(N, d)$ denotes the (average) probability that applying the $N$ th random contact point cages the sphere, while it still was free for $N-1$ contacts. Clearly,

$$
P(N, d)=C(N-1, d)-C(N, d) .
$$

Therefore

$$
\begin{aligned}
\langle N\rangle_{d} & =\sum_{N=1}^{\infty}\left[A(N-1, d) 2^{-(N-1)}-A(N, d) 2^{-N}\right] N \\
& =\sum_{N=0}^{\infty} A(N, d) 2^{-N} .
\end{aligned}
$$

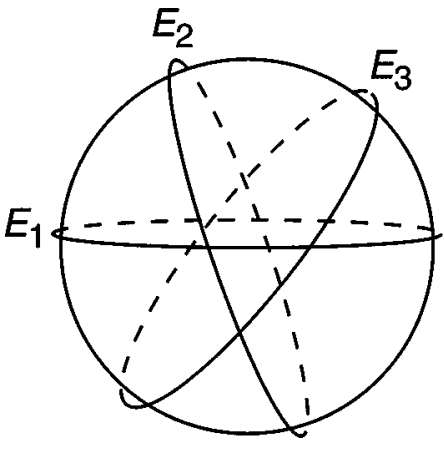

FIG. 3. Each great circle $E$, generated by a contact, intersects all other great circles twice. The line segment between two intersections divides an existing surface sector into two parts.

Equation (4) reduces the caging problem for $N$ contacts to the evaluation of the number $A(N, d)$ of surface sectors produced by these contacts. For the case $d=3$ this evaluation is as follows.

\section{SPHERE CAGING IN $d=3$}

Place $N$ contacts at random on sphere $S^{2}$, or equivalently: draw at random $N$ equators (or great circles) on $S^{2}$ (Fig. 3). As stated before, the probability that two equators coincide is zero. This also applies to equator $E_{N+1}$ of the next contact $c_{N+1}$, which has to intersect all existing $N$ equators. Since intersecting circles intersect each other twice [26] (for two great circles on $S^{2}$ in two diametrically opposite points) it follows that addition of contact $c_{N+1}$ produces $2 N$ new intersections. While connecting these $2 N$ intersections, $E_{N+1}$ also crosses $2 N$ times a surface sector, thereby dividing $2 N$ surface sectors into two parts and thus creating $2 N$ new surface boundaries. In short, the equator $E_{N+1}$ generates $2 N$ additional surface sectors, so the number of surface sectors follows from the recursion relation

$$
A(N+1,3)=A(N, 3)+2 N \text { for } \quad N \geqslant 1 .
$$

Note that $N=0$ corresponds to one surface sector, whereas $N=1$ creates two sectors:

$$
A(0,3)=1, \quad A(1,3)=2
$$

Using the second condition as the initial condition for Eq. (5), we find

$$
A(N, 3)=N^{2}-N+2 \text { for } N \geqslant 1 .
$$

Substitution of the numbers of surface sectors in Eq. (4) finally yields

$$
\langle N\rangle_{3}=1+\sum_{N=1}^{\infty}\left(N^{2}-N+2\right) 2^{-N}=7 .
$$

This is precisely the result obtained earlier by numerical simulation $[23,24]$. 


\section{CAGING PROBABILITY IN $\boldsymbol{d}$ DIMENSIONS}

In $d$ dimensions the calculation is essentially the same as for $d=3$. Each contact point generates a $(d-1)$-dimensional equator (hyper)plane that cuts the $d$-dimensional sphere $(d$ sphere) into two $d$-dimensional hemispheres. (Note that the intersection of the $d$ sphere and the equator (hyper)plane is a $d-1$ sphere.) If we place $N$ contacts on the $d$ sphere, its $d$ -1 dimensional surface $S^{d-1}$ is then divided by the $N$ associated equator (hyper)planes into (by definition) $A(N, d)$ surface sectors. Now add an $(N+1)$ th contact point and its associated equator (hyper)plane. The intersection of the equator (hyper)plane with the $d$ sphere creates a $d-1$ sphere, which is part of the $d$ sphere's surface. By definition, the $N$ other equator (hyper)planes cut this $d-1$ sphere into $A(N, d-1)$ surface parts. These $A(N, d-1)$ parts form new boundaries in the $d$-sphere surface sectors themselves. (Compare to the $d=3$ case, where the two-dimensional equator circle segments formed $2 N$ new boundaries in $2 N$ surface sectors of the 3 sphere). These $A(N, d-1)$ new surface sector boundaries on the $d$ sphere therefore cut $A(N, d-1)$ of the total of $A(N, d) d$-sphere surface sectors in two, so that, by placing the extra contact, the number of surface sectors of the $d$-sphere increases by $A(N, d-1)$. This gives the recurrence relation

$$
A(N+1, d)=A(N, d)+A(N, d-1) \text { for } N>1 .
$$

The initial values are found as follows. A nondivided $d$-dimensional sphere consists of one surface sector. A onedimensional sphere consists of two points (two surface sectors), no matter how many times it is cut in two. Therefore,

$$
\begin{gathered}
A(0, d)=1, \\
A(N, 1)=2 \text { for } N>0 .
\end{gathered}
$$

For small $N$ values $(N \leqslant d), A(N, d)$ has the trivial value $2^{N}$. Up to $N=d$ the addition of an extra contact point means probing an extra spatial dimension, so that all existing equator planes are cut in two parts. For higher $N$ values this is no longer the case; $A(N, d)$ then becomes smaller than $2^{N}$, and one has to rely on Eq. (9). Using relation (9), multiplying both sides by $2^{-N}$, summing from one to infinity, and substituting expression (4) gives

$$
\begin{aligned}
\langle N\rangle_{d} & =\langle N\rangle_{d-1}+2 A(0, d) \\
& =\langle N\rangle_{d-1}+2
\end{aligned}
$$

This single recurrence relation for $\langle N\rangle_{d}$ can easily be solved by using, as the initial value,

$$
\langle N\rangle_{1}=\sum_{N=0}^{\infty} A(N, 1) 2^{-N}=1+\sum_{N=1}^{\infty} 2 \times 2^{-N}=3 .
$$

The final result is

$$
\langle N\rangle_{d}=2 d+1
$$

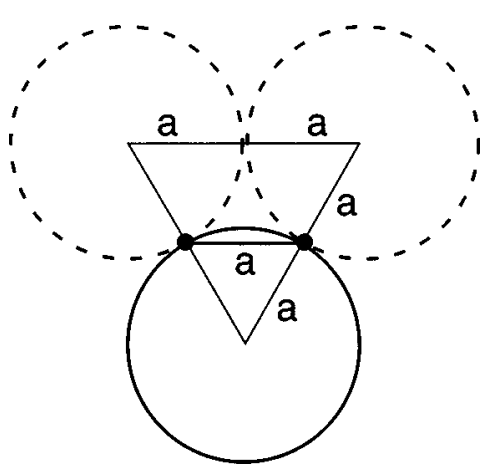

FIG. 4. The shortest distance between contact points in the case of touching spheres is equal to the sphere radius $a$.

for the average number of random contact points needed to cage a sphere in $d$ dimensions.

\section{NUMERICAL SOLUTIONS OF SPHERE CAGING}

We checked the result for $C(N, d)$ [Eq. (1)] by numerical simulations. To obtain approximate values for $C(N, d)$, many random configurations of $N$ points on a $d$-dimensional unit sphere were generated, and the noncaging fraction of them was determined as follows.

A random configuration is constructed by choosing $N$ random points on the surface of the $d$ sphere. Then using all possible sets of $d-1$ points out of these $N$ points, all possible $(d-1)$-dimensional equator (hyper)planes through the sphere center are constructed that contain these $d-1$ points. For each of these (hyper)planes one can easily check whether the remaining $N-d+1$ contact points lie all on one side or on both sides of the hyperplane (by taking the dot product of these $N-d+1$ points with the normal vector of the hyperplane under consideration).

If all $N-d+1$ remaining points are located on one side of the chosen hyperplane, then all $N$ points lie on one hemisphere, and this particular configuration is a free configuration. If the points lie on both sides of the hyperplane, then the chosen configuration is not free with respect to this particular hyperplane, but it may still be free with respect to another hyperplane (which is sufficient for the sphere to be free). If the configuration is not free with respect to any one of all possible hyperplanes, then it is a caging configuration. By repeating this procedure $10^{7}$ times and counting the fraction of free configurations, $A(N, d)$ is calculated. The agreement between the theoretical predictions and the simulation results was found to be excellent (see, for example, Fig. 5).

We also calculated values for the caging probabilities for random configurations of hard spheres (instead of uncorrelated contacts) touching a test sphere. This problem, which is not treated analytically in this paper, is relevant to model the effect of contact correlations which inevitably are present in real hard-sphere systems.

These simulations were performed in the same way as described above, except that the randomly generated contact points on the surface are now regarded to be contacts of the central sphere with constraining spheres, having the same radius as the central sphere. Figure 4 illustrates that in this 
TABLE I. Simulation results of sphere caging.

\begin{tabular}{|c|c|c|c|c|c|c|c|}
\hline$d$ & $N$ & $\begin{array}{l}\text { No. free } \\
\text { confor- } \\
\text { mations }\end{array}$ & $\begin{array}{l}\text { Total no. } \\
\text { confor- } \\
\text { mations }\end{array}$ & $\begin{array}{c}C(N, d) \\
\text { sphere } \\
\text { contacts }\end{array}$ & $\begin{array}{c}C(N, d) \\
\text { point } \\
\text { contacts }\end{array}$ & $\begin{array}{c}\langle N\rangle \\
\text { sphere } \\
\text { contacts }\end{array}$ & $\begin{array}{c}\langle N\rangle \\
\text { point } \\
\text { contacts }\end{array}$ \\
\hline \multirow[t]{3}{*}{2} & 2 & - & - & $1^{\mathrm{a}}$ & $1^{\mathrm{a}}$ & & \\
\hline & 3 & 3406591 & 10000000 & 0,3407 & 0,7500 & & \\
\hline & 4 & 0 & 10000000 & $0^{\mathrm{a}}$ & 0,5000 & 3,34 & 5 \\
\hline \multirow[t]{6}{*}{3} & 3 & - & - & $1^{\mathrm{a}}$ & $1^{\mathrm{a}}$ & & \\
\hline & 4 & 5997315 & 10000000 & 0,5997 & 0,8750 & & \\
\hline & 5 & 1794911 & 10000000 & 0,1795 & 0,6875 & & \\
\hline & 6 & 142701 & 10000000 & 0,0143 & 0,5000 & & \\
\hline & 7 & 562 & 10000000 & 0 & 0,3438 & & \\
\hline & 8 & - & - & $0^{\mathrm{b}}$ & 0,2268 & 4,79 & 7 \\
\hline \multirow[t]{8}{*}{4} & 4 & - & - & $1^{\mathrm{a}}$ & $1^{\mathrm{a}}$ & & \\
\hline & 5 & 7708936 & 10000000 & 0,7709 & 0,9375 & & \\
\hline & 6 & 4079862 & 10000000 & 0,4080 & 0,8125 & & \\
\hline & 7 & 1383658 & 10000000 & 0,1384 & 0,6563 & & \\
\hline & 8 & 271906 & 10000000 & 0,02719 & 0,5000 & & \\
\hline & 9 & 12137 & 4500000 & 0,00270 & 0,3633 & & \\
\hline & 10 & 10 & 150000 & 0,00007 & 0,2537 & & \\
\hline & 11 & - & - & $0^{\mathrm{b}}$ & 0,1719 & 6,35 & 9 \\
\hline \multirow[t]{9}{*}{5} & 5 & - & - & $1^{\mathrm{a}}$ & $1^{\mathrm{a}}$ & & \\
\hline & 6 & 8742127 & 10000000 & 0,8742 & 0,9688 & & \\
\hline & 7 & 6083073 & 10000000 & 0,6083 & 0,8906 & & \\
\hline & 8 & 3245033 & 10000000 & 0,3245 & 0,7734 & & \\
\hline & 9 & 389323 & 3000000 & 0,1298 & 0,6367 & & \\
\hline & 10 & 382818 & 10000000 & 0,03828 & 0,5000 & & \\
\hline & 11 & 16377 & 2000000 & 0,00819 & 0,3770 & & \\
\hline & 12 & 252 & 200000 & 0,0013 & 0,2744 & & \\
\hline & 13 & - & - & $0^{\mathrm{b}}$ & 0,1938 & 7,98 & 11 \\
\hline \multirow[t]{9}{*}{6} & 6 & - & - & $1^{\mathrm{a}}$ & $1^{\mathrm{a}}$ & & \\
\hline & 7 & 2799581 & 3000000 & 0,9332 & 0,9844 & & \\
\hline & 8 & 2270544 & 3000000 & 0,7568 & 0,9375 & & \\
\hline & 9 & 1540468 & 3000000 & 0,5135 & 0,8555 & & \\
\hline & 10 & 860823 & 3000000 & 0,2869 & 0,7461 & & \\
\hline & 11 & 395147 & 3000000 & 0,1317 & 0,6230 & & \\
\hline & 12 & 99241 & 2000000 & 0,0496 & 0,5000 & & \\
\hline & 13 & 7674 & 500000 & 0,0153 & 0,3872 & & \\
\hline & 14 & - & - & $0^{\mathrm{b}}$ & 0,2905 & 9,69 & 13 \\
\hline
\end{tabular}

${ }^{\text {aTheoretical value }}$

${ }^{\mathrm{b}}$ Estimated value; the resulting error in $\langle N\rangle$ is smaller than 0.01 .

case the shortest distance between different contact points is equal to the sphere radius. In the simulations this condition is fulfilled by generating new, random configurations of the $N$ points until the shortest distance between all contact points is larger than or equal to the radius of the unit sphere. Then the simulation is continued as described above. The results are given in Table I.

Figure 5 compares the average cage size for hard-sphere constraints to the analytical result [Eq. (15)] for random point contacts. The hard-sphere cage size (which increases nearly linearly with the dimension $d$ ) is at a given $d$ always smaller than the cage size for random points (which increases exactly linear with $d$ ). This can be understood from the inefficiency of random point constraints to cage a sphere: any correlation which increases the average distance between the contacts will increase their caging probability. A hard sphere attached to contacts is such a "repulsive" correlation.

One could maximize the effect of such correlations by requiring that the distance between any pair of constraints on a test sphere must be maximal. (For an extensive discussion of such maximization problems, see Ref. [27].) This would be a way to find the minimum number of constraints needed to cage a sphere. For example, an equilateral triangle $(N$ $=3$ ) cages a sphere in $d=2$, and a tetrahedron $(N=4)$ cages a 3D sphere. This already suggests that

$$
N_{\min d}=d+1
$$




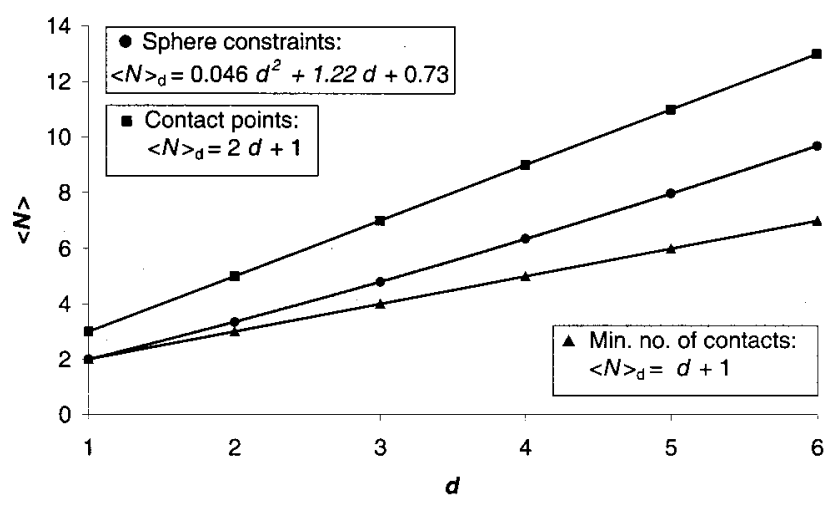

FIG. 5. Simulation results for the average number of contacts $\langle N\rangle_{d}$ needed to cage a sphere in $d$ dimensions. The caging occurs by random point contacts or by spherical constraints. (For $d=1$, both data points are theoretical values.)

is the minimal size of a cage for arbitrary dimension. Indeed, Eq. (16) follows from the fact that $d$ contacts form a number of surface sectors given by: $A(d, d)=2^{d}$. Then the probability $C(d, d)$ in Eq. (1) equals unity. As explained near Eq. (11), $A(d+1, d)<2^{d+1}$, so that $C(d+1, d)<1 \quad(d+1, d)$ $<1$. Therefore one can always construct a caging configuration with $d+1$ contacts. A consequence of Eqs. (15) and (16) is that the average cage size due to repulsive contacts must satisfy

$$
d+1 \leqslant\langle N\rangle_{d} \leqslant 2 d+1 .
$$

Our simulations for hard-sphere contacts in Fig. 5 comply with this requirement. Figure 6 compares analytical and simulation results for the caging probability for a sphere in three dimensions.

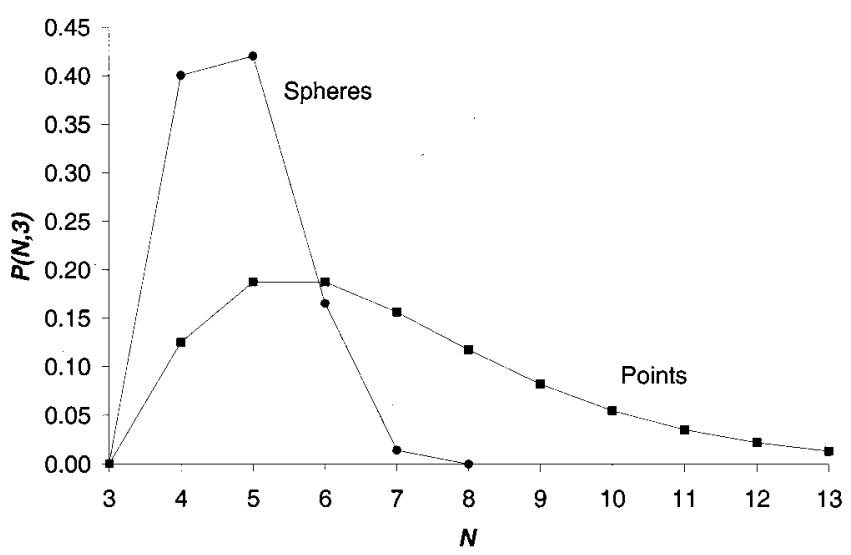

FIG. 6. Probability $P(N, 3)$ that a sphere becomes caged in three dimensions on placing the $N$ th random constraint. The simulation results (-) for spherical constraints show that a test sphere is very likely caged by a number of hard spheres in the range $4-7$. The analytical results (ם) show that uncorrelated point contacts are much less effective "cage formers.' (Lines are drawn to guide the eye.)
For spherical constraints both the increase and decay of the probability is much more pronounced than for random contacts. Note that for random points there is, of course, no limit to the number of contacts $N$ on a free, uncaged sphere. For hard spheres there is obviously a maximum value. For $d=1,2$, and 3 there is a maximum number of, $N=1,4$, and 9 spheres, respectively, which may contact a test sphere without caging it (the maximum apparently equals $d^{2}$ ). The 3D maximum concerns a triangle of three spheres which "support" the test sphere such that only one free direction vector (perpendicular to the triangle) is left. Then a hexagon of six spheres can be added which all leave the direction vector free. However, the next contact $N=10$ always closes the cage. Therefore, in Fig. $6, P(N=3)=0$ for $N \geqslant 10$. For $3<N<10$, the probability is finite, but it is clear from Fig. 6 that random sphere cages larger than 8 are rare events. These predictions are confirmed by computer generated contact distributions for 3D random sphere packings [28]. Interestingly, 10 is the largest contact number observed [28], realized only by an extremely small fraction of spheres.

\section{COMPARISON WITH RANDOM SPHERE PACKINGS}

In a random dense sphere packing (RDP) the majority of spheres is arrested by its neighbors. If this arrest implies absence of any free direction vector, the majority of spheres in a RDP is caged, according to definitions in Sec. II. Therefore, we have re-examined coordination numbers in experimental and simulated hard-sphere packings from a caging point of view, in particular because there seems to be no unanimous agreement on the value (and physical meaning) of these coordination numbers [4-21].

Often, neighboring spheres that do not touch the test sphere are still included in reported coordination numbers. However, we adopt the view that only neighbor spheres that are in real contact with a test sphere form its constraining cage. Therefore, comparison with literature results should be made with care, as one needs to specify a minimal distance between two surfaces below which the surfaces are regarded to be in "real" contact. Variations in this cutoff distance and its extrapolation to zero give rise to a variety of average coordination numbers $\langle c\rangle$ in the literature [4-21]. Early investigators [7] estimated that $\langle c\rangle \approx 10$. Bernal and Mason [8] arrived at a more realistic average of about 6 , on the basis of experiments as well as the argument that "each sphere may be considered in general to rest on three others and in turn supports another three", [8]. Some authors [29] even used $\langle c\rangle=6$ as the criterion for choosing the "correct" cutoff distance of 1.057 sphere diameters, which is certainly too large in view of Fig. 7, as discussed below. Bennett [15] also argued that mechanical stability requires $\langle c\rangle=6.0$, and also found this value by extrapolation from his simulation data. This extrapolation, however, is not quite straightforward, and, moreover, the simulated packing densities $(\phi=0.61)$ are below the experimental value of $\phi=0.64$. This suggests that Bennett's sphere deposition technique [15] does not reproduce all details of a real RDP. Such a shortcoming was also noted in other simulation studies $[14,16]$ where mean 


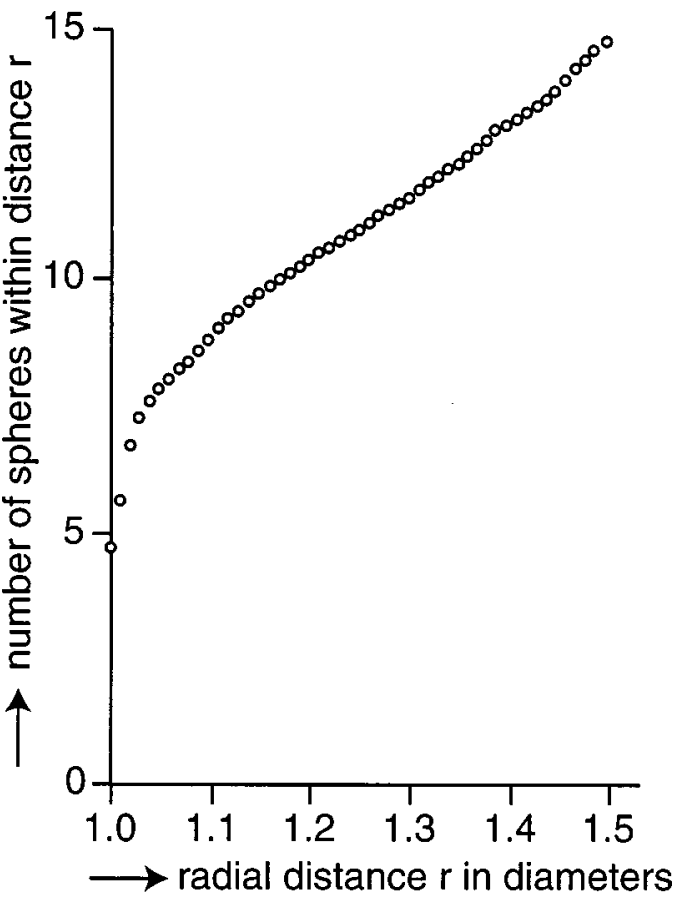

FIG. 7. Number of neighbor spheres within a radial distance $(r)$ of the central sphere according to Mason [11] obtained from experiments of Scott [9] on large numbers of randomly packed, smooth steel balls. (Sphere positions were determined with an accuracy better that $1 \%$ of their diameter [9].) Note the steep gradient near the contact number at $r=1.0$.

nearest neighbor numbers close to 6 were also found, though the physical justification was reported to be unclear. Goto and Finney [17] used $\langle c\rangle=6.0$ as input for their calculation of the RDP density. They admitted that this number is still open to argument. Indeed, Goto and Finney [17] depicted a nonlinear extrapolation to zero cutoff distance which does not exclude lower coordination numbers $\langle c\rangle<6$.

This nonlinearity was clearly shown and emphasized by Mason [11], who reanalyzed the original data from extensive experiments of Scott [9] on the radial distribution of spheres in a RDP. The outcome of Mason's analysis (see Fig. 7) clearly shows the steep gradient in the experimental contact number when sphere surfaces are very close together. Mason confirmed Scott's finding that there are $9.3 \pm 0.8$ neighbors within 1.1 diameters from the center of a test sphere (Fig. 7). However, he concluded [11] that on average there are only about five actual contacts. Since Scott [9] reported an accuracy of better than $1 \%$ in the radial distance measurements, this conclusion is justified. The experimental coordination number determined from Fig. 7 is actually $4.76 \pm 0.02$, which agrees well with our simulation result of $\langle N\rangle_{3}=4.79 \pm 0.02$. Note that the contact number (i.e., the coordination number at $r=1.0)$ in Fig. 7 is in any case sandwiched between the minimal value of $d+1=4$ and the cage size $2 d+1=7$ for random contacts [Eq. (17)].

\section{DISCUSSION}

We have found that the average experimental coordination number of a RDP obtained from proper extrapolation
(Fig. 7) equals the average cage size for an individual test sphere contacted by hard spheres. This is an interesting result because it indicates that the coordination number is determined by only two basic features of a RDP, namely, that the majority of spheres is arrested (caged) and that the spheres cannot interpenetrate (the volume exclusion which produces the contact correlations).

This interpretation of the physical origin of the (value of) the coordination number implies that the random sphere packing is basically the same as the random caging of spheres by spherical constraints. Therefore the RDP volume fraction $\phi \approx 0.64$ seems in a sense an accidental consequence of caging effects, rather than the result of some maximization procedure for the density or coordination numbers. A caged test sphere could still in some cases accommodate additional spheres in its "coordination shell" of contacting neighbors (consider, for example, a sphere caged by only four others). However, this accommodation may be obstructed by the fact that the majority of the spheres in the vicinity of the caged test sphere are immobilized as well. Therefore, we expect that the coordination number (and the corresponding density) in a real RDP cannot rise much above the average size (Fig. 5) of a random hand-sphere cage (and the corresponding density).

Our caging analysis also makes clear why the coordination number in a RDP must be a distributed quantity. This is inherent to the statistics of the cage size (Fig. 6). Note the strong difference from the hexagonal close sphere packing which maximizes the density for a single-valued coordination number of $c=12$.

Whether our analysis ultimately implies that random close sphere packings can be generated quantitatively by a local caging rule is still an open question. If such a rule applies, one would expect, on the basis of Fig. 6, that the tail of the contact distribution does not extend much above $c=7$ or 8 . This is indeed the trend observed in various studies [21,28] on random sphere packings.

Finally it should be noted that in the simulation of sphere constraints in Fig. 6, spheres are fixed after being placed in contact with the test sphere. In the experiments of Scott [9], however, a RDP is formed by pouring and shaking so that (groups of) spheres may reorganize. Perhaps this difference in mobility affects the comparison of contact numbers. The extensive computer simulations of Ref. [28], for a very small cutoff distance of $10^{-7}$ sphere diameters, yielded an average contact number of $\langle c\rangle=5.8295$, consistent with Eq. (17), but larger than expected from Fig. 7. It remains to be investigated how contact numbers depend on details of the construction of a random sphere packing by either experiments, simulations $[28,29]$, or application of a simple caging rule. What is clear, nevertheless, is that the widely quoted value of $\langle c\rangle=6.0[21,30]$ very likely represents an underestimation of the efficiency with which hard spheres cage each other in random dense packings.

\section{CONCLUSIONS}

The average number of randomly placed point constraints needed to cage a $d$-dimensional sphere is equal to $\langle N\rangle_{d}$ 
$=2 d+1$. To prove this result, configurations of contact points can be grouped into equivalent subsets (called reflection sets), from which caging probabilities can be deduced without a need for averaging over all possible configurations. Simulations of the caging by spherical constraints show that hard spheres are much more effective "cage formers" than random contacts. The simulation value of $\langle N\rangle_{3}=4.79$ $( \pm 0.02)$ agrees with the experimental average coordination number (of 4.76 \pm 0.02 ) in the random dense sphere packing, according to Mason [11]. This result supports the physical picture that the coordination number in random close packings of spheres is foremost determined by the individual sphere mobility and caging behavior. This result suggests that RDP properties might well be derived from the (local) caging behavior of individual spheres.

\section{ACKNOWLEDGMENTS}

M. Lanen, M. Uit de Bulten, and I. Van Rooijen are acknowledged for their help and patience in preparing the manuscript. G. Koenderink and W. Kegel are thanked for stimulating discussions. J. K. G. Dhont is thanked for his critical comparison of earlier versions of the theory. The EC Colloid Physics Network meeting in Varenna 1998 initiated collaboration between A.P. and M.K.
[1] P. N. Pusey, in Liquids, Freezing and Glass Transition, edited by J. P. Hansen, D. Levesque, and J. Zinn-Justin (Elsevier, Amsterdam 1991), p. 899.

[2] P. N. Pusey and W. van Megen, Nature (London) 320, 340 (1986).

[3] J. D. Bernal, Trans. Faraday Soc. 33, 27 (1937).

[4] J. D. Bernal, Nature (London) 183, 141 (1959).

[5] J. D. Bernal and J. Mason, Nature (London) 188, 910 (1960).

[6] J. D. Bernal, Proc. R. Soc. London, Ser. A 280, 299 (1964).

[7] W. O. Smith, P. D. Foote, and P. F. Busang, Phys. Rev. 34, 1271 (1929).

[8] J. D. Bernal and J. Mason, Nature (London) 188, 111 (1960).

[9] G. D. Scott, Nature (London) 194, 956 (1962).

[10] D. P. Haughey and G. S. G. Beveridge, Chem. Eng. Sci. 21, 905 (1966).

[11] G. Mason, Nature (London) 217, 733 (1968).

[12] R. H. Beresford, Nature (London) 224, 550 (1969).

[13] J. L. Finney, Proc. R. Soc. London, Ser. A 319, 479 (1970).

[14] W. M. Visscher and M. Bolsterli, Nature (London) 239, 504 (1972).

[15] C. H. Bennett, J. Appl. Phys. 43, 2727 (1971).

[16] E. M. Tory, B. H. Church, M. K. Tam, and M. Ratner, Can. J. Chem. Eng. 51, 484 (1973).

[17] K. Goto and J. L. Finney, Nature (London) 252, 202 (1974).
[18] G. D. Scott and D. M. Kilgour, Br. J. Appl. Phys. 2, 863 (1969).

[19] J. D. Bernal, I. A. Cherry, J. L. Finney, and K. R. Knight, J. Phys. E 3, 388 (1970).

[20] D. J. Cumberland and R. J. Crawford, The Packing of Particles (Elsevier, Amsterdam, 1987).

[21] Disorder and Granular Media, edited by D. Bideau and A. Hansen (North-Holland, Amsterdam, 1993).

[22] A. P. Philipse and A. Verberkmoes, Physica A 235, 186 (1997).

[23] S. G. J. M. Kluijtmans, Ph.D. thesis, Utrecht University, 1998.

[24] A. P. Philipse and S. G. J. M. Kluijtmans, Physica A 274, 516 (1999).

[25] D. A. Brannan, M. F. Esplen, and J. J. Gray, Geometry (Cambridge University Press, Cambridge, 1999)

[26] Euclid, The Elements, Book III, translated by S. L. Heath (Dover, New York, 1956).

[27] H. Melissen, Packing and Covering with Circles, Ph.D. thesis, Utrecht University, 1998.

[28] B. D. Lubachevsky, F. H. Stillinger, and E. N. Pinson, J. Stat. Phys. 64, 501 (1991).

[29] A. S. Clarke and H. Jónsson, Phys. Rev. E 47, 3975 (1993).

[30] Advances in the Mechanics and Flow of Granular Materials, edited by M. Shahinpoor (Gulf Publishing Company, Houston, 1983), Vol. 1. 